\title{
Does stigma impair prevention of mental disorders?
}

\author{
Nicolas Rüsch and Graham Thornicroft
}

\section{Summary}

Prevention of mental disorders can be effective, but is rarely implemented in routine settings. Here we propose a matrix to show how different aspects of stigma, discrimination and lack of knowledge can hinder different types of prevention, including early intervention. Programmes to reduce stigma's impact and so to facilitate prevention are needed.

\section{Declaration of interest}

None.
Nicolas Rüsch (pictured) is a consultant psychiatrist and Professor of Public Mental Health at the Department of Psychiatry II, University of UIm, Germany. Graham Thornicroft is a consultant psychiatrist at the South London \& Maudsley NHS Foundation Trust, and Professor of Community Psychiatry, King's College London, Institute of Psychiatry, UK.

The potential benefits from preventing mental illnesses are considerable, both in terms of illness burden for people with mental illness, their families and carers as well as in socioeconomic terms. Evidence so far suggests that prevention can reduce the incidence of mental disorders and can be cost-effective. ${ }^{1}$ However, available prevention strategies are rarely implemented in routine public health or mental health service programmes. Here we put forward the hypothesis that the stigma and discrimination associated with mental illness may act as barriers to preventing mental illnesses. We propose a $4 \times 3$ matrix to show how three types of stigma and lack of knowledge can hinder three types of prevention (see Appendix).

\section{Forms of prevention and stigma}

Prevention in mental health can be categorised in two ways. ${ }^{2}$ The traditional classification distinguishes prevention according to the illness stage: primary prevention addresses risk factors in order to reduce the number of new cases or the incidence; secondary prevention occurs during the early stages of the disease process; and the domain of tertiary prevention is relapse prevention and rehabilitation after illness onset. The Institute of Medicine's ${ }^{3}$ newer classification arranges prevention efforts according to the target group: universal prevention in the general population, selective prevention for persons at risk (for example children of parents with mental illness), and indicated prevention for people with early signs and symptoms who do not meet diagnostic criteria, but who are at high risk to be in the early subthreshold stages of a developing mental illness. In the Appendix we collapse selective, indicated and secondary prevention into one category, referring to prevention for individuals at elevated risk to develop a mental illness as compared with the general population.

Stigma associated with mental illness is an overarching term that comprises three main components: negative stereotypes (such as 'people with mental illness are responsible for their condition'); prejudice that refers to agreeing with these stereotypes and negative emotional reactions ('That's right, they are to blame and I am angry at them'); and finally discrimination as the behavioural consequence ('I oppose funding for mental health prevention'). Lack of knowledge or poor mental health literacy is a distinct, but related concept insofar as negative stereotypes about people with mental illness represent factually wrong or one-sided assumptions that are commonly held in society and are a prerequisite for stigma. However, mental health literacy is more than the absence of erroneous stereotypes and includes the recognition of a developing disorder as well as knowledge about prevention and effective interventions. ${ }^{4}$ We therefore refer to poor knowledge as a barrier to prevention in its own right in addition to stigma.

Stigma can express itself in three ways: (a) public stigma, when members of the general public endorse prejudice and discrimination against people with mental illness; (b) self-stigma when people with mental illness agree with and internalise negative stereotypes, leading to low self-esteem, shame, demoralisation and giving up life goals; ${ }^{5}$ and (c) structural discrimination or rules and regulations in society that intentionally or unintentionally disadvantage people with mental illness, for example by the amount of resources dedicated to mental health services.

\section{Stigma and lack of knowledge as barriers to prevention}

In the Appendix, we outline how three forms of stigma as well as poor mental health literacy can impair three types of prevention. We postulate the following two main effects. First, prevention may simply not happen because it is not implemented (because of structural discrimination, public stigma or lack of knowledge) or because people do not participate in it (avoidance as a result of fear of public stigma, self-stigma or lack of knowledge). Second, even if implemented, prevention may not succeed (because of direct negative effects of public, self or structural stigma on well-being of the target group). More specifically and regarding different types of prevention, primary or universal prevention will likely be more difficult to implement among members of the public who have poor knowledge about mental health, who distance themselves from people with mental illness, who hold pessimistic views about the benefits of prevention, and who are opposed to channelling resources towards mental health. ${ }^{4,6}$ With respect to tertiary prevention, on the other hand, there is ample qualitative and quantitative evidence that stigma is a barrier to mental health service use and relapse prevention. ${ }^{7}$ Conceptually and practically, secondary prevention has an intermediate status because its target population, people at higher risk to develop a mental disorder, may or may not self-label and refer to themselves as having a mental health problem. Depending on their self-concept and the type and timing of the intervention, public stigma and selfstigma/shame may hinder them to participate or may, for example because of stigma as a stressor, worsen clinical outcomes. 


\section{Applying the stigma-prevention matrix to future research}

The matrix may serve several purposes. First of all, the literatures on prevention and on stigma, both rich in their own right, could be better connected to allow more cross-fertilisation. For example regarding the potential role of stigma for indicated prevention among young people at risk for psychosis, recent findings suggest that the more young people at risk perceive stigma as a stressor that exceeds their coping resources, the more negative their attitudes are towards seeking professional help. ${ }^{8}$ Another example is that beyond attitude surveys we do not know what members of the public actually do to prevent mental disorders, let alone how their attitudes and knowledge are related to their real-world behaviours. ${ }^{4}$ A final example is that public surveys suggest that although members of the public recognise the burden and disability caused by mental disorders they remain hesitant to allocate resources to mental health prevention and services, but the role of stigma for public preferences is still poorly understood. ${ }^{6,9}$

Second, our matrix might be used to generate and test hypotheses on how interventions meant to address stigma or lack of mental health-related knowledge affect prevention, and vice versa how prevention efforts influence stigma variables. For instance, interventions reducing the shame and self-stigma associated with at-risk state or early psychosis could increase the success of prevention because, by addressing shame and self-stigma, such programmes target factors that undermine self-efficacy and hope. On the other hand, selective or indicated prevention may well lead to intended or unintended (self-)labelling as 'mentally ill'. This label may be helpful, facilitating help-seeking, or harmful, leading to stigma and self-stigma. ${ }^{8,10}$ Another example refers to the allocation of resources to the prevention of mental disorders as an example of unintended structural discrimination. Studies are needed to examine whether interventions targeting policy makers or the general population will achieve a fairer resource allocation and improve the implementation and quality of prevention programmes.

Once we better understand the interaction of knowledge, stigma and prevention, we will be able to design and implement interventions for both sides of the coin: prevention programmes in public health and clinical settings as well as addressing the social consequences of mental illness. This two-pronged approach, taking into account the illness and the stigma associated with it, is needed to reduce the burden of mental illness on individuals and society.

Nicolas Rüsch, MD, Department of Psychiatry II, University of UIm, Germany, and Health Service and Population Research Department, King's College London, Institute of Psychiatry, UK; Graham Thornicroft, MD, PhD, Health Service and Population Research Department, King's College London, Institute of Psychiatry, UK

Correspondence: Nicolas Rüsch, Section Public Mental Health, Department of Psychiatry II, University of UIm, Parkstrasse 11, D-89073 UIm, Germany. Email: nicolas.ruesch@uni-ulm.de

First received 6 May 2013, final revision 12 Nov 2013, accepted 28 Nov 2013

\section{Appendix}

\section{Matrix of knowledge and stigma variables (four rows in the lower part of the table) and how they may hinder different types of prevention (three columns)}

Traditional classification of

prevention according to illness phase

New classification of prevention according to target population Examples

Poor knowledge

Public stigma

Self-stigma

\author{
Primary prevention
}

Universal prevention

- Media campaigns against alcohol misuse or violence in the family
- Poor knowledge about early and protective factors

- Unwillingness to participate in or implement prevention due to prejudice against people with mental illness

- Pessimism about success of prevention

\footnotetext{
- Allocation of fewer resources to prevention in mental health

- Negative media portrayals of people with mental illness that discourage prevention
}

Secondary prevention

Selective or indicated prevention

- Group programmes for children of parents with mental illness (selective prevention)

- Early intervention for young people at risk for psychosis (indicated prevention) intervention

Tertiary prevention

$$
\begin{aligned}
& \text { - Relapse prevention } \\
& \text { - Low threshold services for people } \\
& \text { with mental illness currently not } \\
& \text { in treatment } \\
& \text { - Poor knowledge about available } \\
& \text { treatments and relapse prevention } \\
& \text { - Avoidance of service use due to } \\
& \text { fear of public stigma } \\
& \text { - Discrimination as a barrier to } \\
& \text { recovery (for example work, } \\
& \text { relationships, housing) } \\
& \text { - Avoidance of service use because } \\
& \text { of self-stigma/shame } \\
& \text { - Demoralisation, 'why try', and } \\
& \text { social isolation as consequences } \\
& \text { of self-stigma } \\
& \text { - Under-resourced mental health } \\
& \text { services lead to poorer quality } \\
& \text { and quantity of available mental } \\
& \text { health services }
\end{aligned}
$$
- Under-resourced mental health services lead to poorer quality and quantity of available mental
- Avoidance of service use due to fear of public stigma
- Discrimination as a barrier to recovery (for example work relationships, housing) of self-stigma/shame
- Demoralisation, 'why try', and social isolation as consequences

- Poor recognition of early signs and symptoms

- Avoidance of early recognition/ stigma

- Pessimism about success of early intervention

- Labelling as unintended consequence of prevention, leading to stigma-related stress

- Avoidance of early recognition/ intervention because of selfstigma/shame

- Self-labelling as unintended consequence of prevention, leading of self-stigma to shame and demoralisation

- Underresourced early intervention services 


\section{References}

1 Mihalopoulos C, Vos T, Pirkis J, Carter R. The economic analysis of prevention in mental health programs. Annu Rev Clin Psychol 2011; 7: 169-201.

2 Compton MT. Clinical Manual of Prevention in Mental Health. American Psychiatric Publishing, 2010.

3 Institute of Medicine. Reducing Risks for Mental Disorders: Frontiers for Preventive Intervention Research. National Academy Press, 1994.

4 Jorm AF. Mental health literacy: empowering the community to take action for better mental health. Am Psychol 2012; 67: 231-43.

5 Corrigan PW, Larson JE, Rüsch N. Self-stigma and the "why try" effect: impact on life goals and evidence-based practices. World Psychiatry 2009; 8: 75-81.

6 Schomerus G, Matschinger $\mathrm{H}$, Angermeyer MC. Preferences of the public regarding cutbacks in expenditure for patient care: are there indications of discrimination against those with mental disorders? Social Psychiatry Psychiatr Epidemiol 2006; 41: 369-77.

7 Clement S, Schauman O, Graham T, Maggioni F, Evans-Lacko S, Bezborodovs $N$, et al. What is the impact of mental-health related stigma on help-seeking? A systematic review of quantitative and qualitative studies. Psychol Med, 2014; in press.

8 Rüsch N, Heekeren K, Theodoridou A, Dvorsky D, Müller M, Paust T, et al. Attitudes towards help-seeking and stigma among young people at risk for psychosis. Psychiatry Res 2013; 210: 1313-5.

9 Corrigan PW, Watson AC. Factors that explain how policy makers distribute resources to mental health services. Psychiatr Serv 2003; 54: 501-7.

10 Rüsch N, Corrigan PW, Heekeren K, Theodoridou A, Dvorsky D, Metzler S, et al. Well-being among persons at risk of psychosis: the role of self-labelling, shame, and stigma-stress. Psychiatr Serv 2014; doi: 10.1176/ appi.ps. 201300169 .

\section{Embracing autistic traits: Spock's Vulcan heritage in Star Trek}

\section{Geoff Lawrence-Smith}

Star Trek has become a modern institution, a franchise spanning decades from the pilot episode in 1966 to J. J. Abrams' 2013 movie instalment, Star Trek: Into Darkness. Amidst its action and excitement, the success of the Star Trek series lies in its often perceptive social commentary.

Amid the tapestry of the Star Trek universe lies the Vulcan civilisation. Vulcans were once a barbarous and chaotic people who embraced ritualistic emotional control and the rigid discipline of logic to prevent their self-annihilation. In doing so, the Vulcans actively embraced characteristics the viewers might consider 'autistic' in order to aid their own survival. Indeed, the stigma Vulcans experience from others within the Star Trek universe parallels the stigma experienced by people with autism spectrum conditions in our own society.

Between the Vulcan and human cultures, the iconic character of Mr Spock strives to identify himself. Aboard the starship Enterprise, spock's continuing journey of self-discovery and understanding mirrors that of his culture's past and forms the backbone to his relationships with colleagues and friends. As Spock's character becomes better understood, he repeatedly demonstrates how his autistic traits serve him well when 'saving' the day.

Many perceive autistic traits in a negative way as rigid thinking, literal interpretation, tactlessness, non-conformity, and a disdain of aimless social interaction. In contrast, the character of Spock demonstrates logical, principled and methodological thinking, making astute appraisals of situations that he communicates to others without the need to obscure his ideas with social nuance and complexity. This is exemplified in Into Darkness, when Spock and Captain Kirk are reprimanded for insubordination.

'Admiral Pike: Are you giving me attitude, Spock?

Spock: I am expressing multiple attitudes simultaneously, sir. To which one are you referring?'

'Admiral Pike: That's a technicality.

Spock: I am Vulcan, sir. We embrace technicalities.'

Spock has always demonstrated an acute and immutable sense of social justice with an astounding eye for detail (especially concerning Starfleet regulations) which he often employs to temper Kirk's rash decision-making in critical situations.

'I can not allow you to do this [Captain]. It is my function aboard the ship to advise you in making the wisest decisions possible, something I firmly believe you are incapable of doing in this moment.' (Into Darkness)

'Nowhere am I so desperately needed as among a shipload of illogical humans.' (Star Trek, the original series)

Although he often misunderstands human motivations, Spock is a highly intelligent, autonomous and original thinker who notices patterns others rarely do. He is also a genuine and loyal friend who is immensely valued by those around him.

Kirk eulogising Spock: 'Of my friend, I can only say this: of all the souls I have encountered in my travels, his was the most . . . human.' (Star Trek: The Wrath of Khan)

Spock could be an all-too-rare positive role model for those with autism spectrum conditions, promoting, as he does, the value of 'autistic' attributes and reframing negative stereotypes of autism in a more positive light. 